\title{
Structural Origin of Dimensional Effect on Dielectric Breakdown Strength of ZnO Vraistors
}

\author{
Non-member Shengtao Li, Fuyi Liu, Guangping Jia (Xi'an Jiaotong University) \\ Member Yoshimichi Ohki (Waseda University)
}

Keywords: Zinc oxide varistor, Dimensional effect, Grain shape, Breakdown strength

Zinc oxide $(\mathrm{ZnO})$ varistors are widely used for transient protection in electronic circuits and electrical systems, since they have a super - nonlinear voltage - current characteristic and high withstanding capability against surge overvoltage. (1), (2) Although it is generally considered that grain boundaries play important roles in the varistor characteristics, the details are unknown. ${ }^{(3)-(5)}$ In the present paper, the effect of the grain shape is discussed based on the experimental results on the thickness dependence of the breakdown strength. Four types of samples were used. Sample $\mathrm{H}$ has a relatively high breakdown field and is a commercial product in a disk shape with a diameter of $7.4 \mathrm{~mm}$ and a thickness of 1 . $98 \mathrm{~mm}$. Sample $\mathrm{M}$ has a medium breakdown filed and is a commercial product in a disk shape with a diameter of 7.4 $\mathrm{mm}$ and a thickness of $0.71 \mathrm{~mm}$. Samples L1 and L2 have a relatively low breakdown field and are made for the present experiments and in a disk shape with a diameter of $14.0 \mathrm{~mm}$ and a thickness of $1.71 \mathrm{~mm}$.

In order to change the sample thickness, the sample was carefully thinned from both sides by grinding. Silver was sintered on both surfaces and used as electrodes. When the DC current flowing through the sample became $1 \mathrm{~mA}$, the voltage across it was measured and taken as a breakdown voltage. After the sample has been polished, preferentially etched in HF solution, cleaned and finally dried, the sample structure was observed with an optical microscope. Since the grain shape is elliptic, the longer and the shorter diameters of each grain were measured.

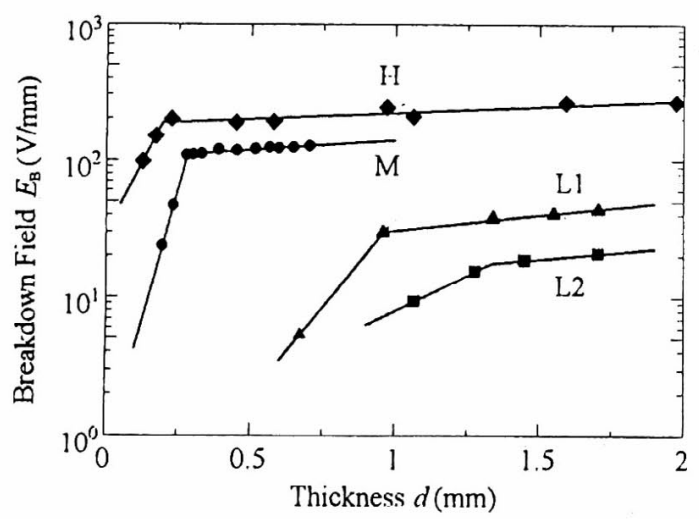

Fig. 1 Thickness dependence of the breakdown field for four types of $\mathrm{ZnO}$ varistors.

The relation between the breakdown field $E_{B}$ and the thickness $d$ of various samples is shown in Fig. 1. It can be seen that there exists a transition point for all the samples, which is characterized by the critical thickness $d_{c}$ and the transition breakdown field $E_{c}$. Before the transition point where $d$ is smaller than $d_{c}$, breakdown field $E_{\mathrm{B}}$ increases sharply with an increase in $d$, while it increases slowly after the transition point. That is to say, if $E_{\mathrm{B}}$ is expressed as

$$
E_{B} \propto \exp (b d) \text {, }
$$

the exponent $b$ before the transition (referred to as $b_{1}$ ) is larger than the one after the transition (referred to as $b_{2}$ ). Table 1 shows the values of $b_{1}, b_{2}, d_{c}$ and $E_{\mathrm{c}}$ for the four types of varistors.

Table 1 The exponents $b_{1}$ and $b_{2}$, the critical thickness $d_{c}$ and the transition breakdown field $E_{c}$ of four types of $\mathrm{ZnO}$ varistors.

\begin{tabular}{|c|c|c|c|c|}
\hline Sample & $b_{1}$ & $b_{2}$ & $d_{c}(\mathrm{~mm})$ & $E_{c}(\mathrm{~V} / \mathrm{mm})$ \\
\hline $\mathrm{H}$ & 9.4 & 0.21 & 0.19 & 185 \\
\hline $\mathrm{M}$ & 18.6 & 0.31 & 0.28 & 110 \\
\hline $\mathrm{L} 1$ & 6.0 & 0.36 & 0.98 & 33 \\
\hline $\mathrm{L} 2$ & 2.4 & 0.43 & 1.34 & 18 \\
\hline
\end{tabular}

From Table 1 , it is clearly seen that $b_{2}$ decreases as $E_{c}$ increases. While $b_{1}$ increases as $E_{c}$ increases except for sample $\mathrm{H}, d_{c}$ clearly decreases as $E_{c}$ increases. Such an effect of $d$ on $E_{B}$ is called dimensional effect. As mentioned above, the sample thickness was changed by grinding in the present experiments. However, one of the authors (G. J. ) has reported

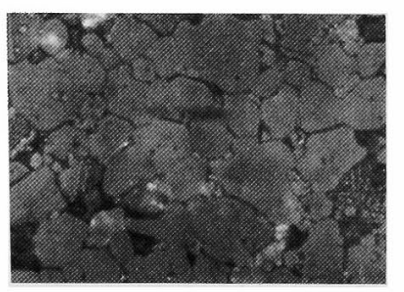

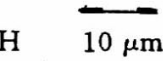

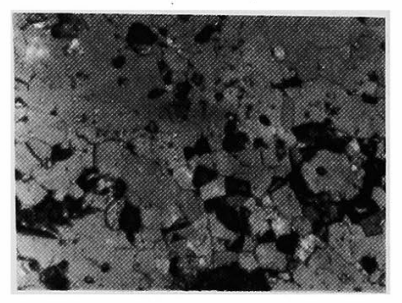

L1 $20 \mu \mathrm{m}$

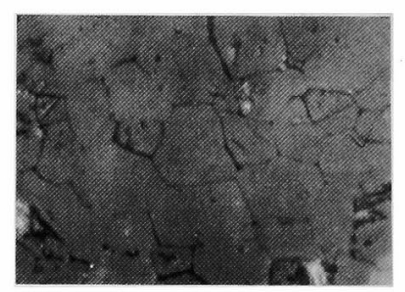

$\mathrm{M} \quad \overline{10 \mu \mathrm{m}}$

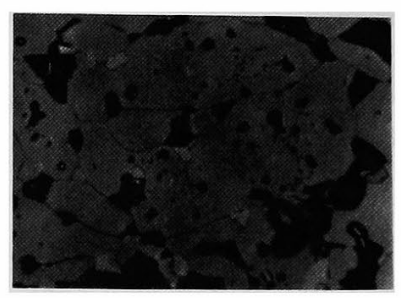

L2 $20 \mu \mathrm{m}$
Fig. 2 Optical micrographs of four types of $\mathrm{ZnO}$ varistors. 
the same tendencies for samples with different as - grown thickness ${ }^{(6)}$. Therefore, it is considered that the dimensional effect is an intrinsic characteristic of $\mathrm{ZnO}$ varistors, and that the values $b_{1}, b_{2}, d_{c}$ and $E_{c}$ are the factors representing the dimensional effect.

Optical micrographs of the samples are shown in Fig2. Sample $H$ has a well-developed microstructure with nearly hexagonal grains of a similar size, and the number of pores is small. In the case of sample $\mathrm{M}$, most grains are of a slightly elongated hexagonal shape and their sizes are scattered to some extent. Compared with samples $\mathrm{H}$ and $\mathrm{M}$, the grains in samples L1 and L2 are elliptic in shape with a highly nonuniform size distribution. It contains a large number of pores.

From the optical micrographs, the longer and the shorter diameters of more than 150 grains were measured for every sample, and their average and standard deviation, and the aspect ratio between the two diameters was calculated. Figure 3 shows the relation between the critical thickness $d_{c}$ and the dispersive ratio between the standard deviation and the average of the longer diameters. It is clearly shown that $d_{c}$ depends linearly on the dispersive ratio along the longer diameter. A similar investigation indicates that $d_{c}$ also depends linearly on the dispersive ratio along the shorter diameter. It is simply concluded that the critical thickness $d_{c}$ is determined by the dispersive ratio of the $\mathrm{ZnO}$ grain size.

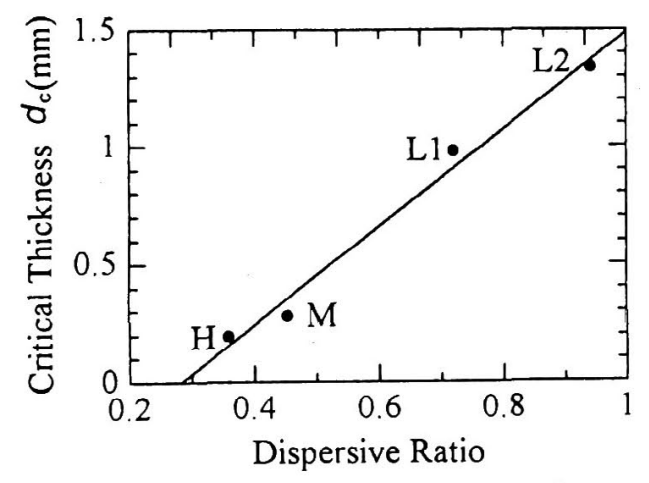

Fig. 3 Relation between the critical thickness $d_{\mathrm{c}}$ and the dispersive ratio along the longer diameter of grains.

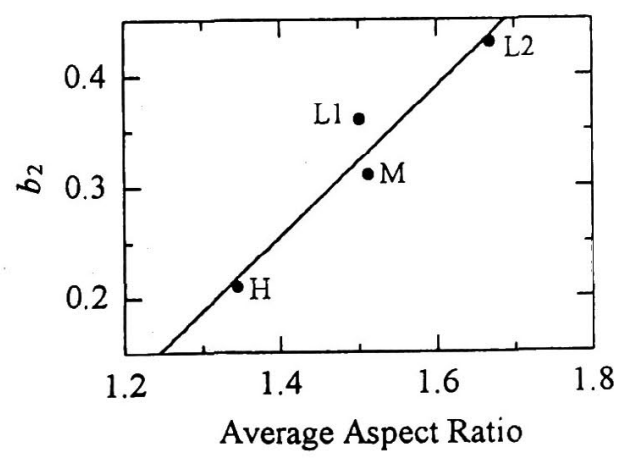

Fig. 4 Relation between $b_{2}$ and the average aspect ratio.

Figure 4 shows the relation between the exponent $b_{2}$ and the aspect ratio. It is shown that $b_{2}$ increases linearly with an increase of the average aspect ratio. This indicates that the thickness dependence of the breakdown field $E_{B}$ is enhanced as the grain shape becomes more elongated.

To conclude, the two statistical parameters, the aspect ratio and the dispersive ratio for the grain shape, are proved to be useful parameters to characterize the dimensional effect and to estimate the dielectric strength of $\mathrm{ZnO}$ varistors. Since the dimensional effect is seen not only in the breakdown field, but also in the nonlinear coefficient and the withstanding capability per unit volume, the present finding is very important.

This work was supported in part by Natural Science Foundation of China (No. 59507005[1996 - 1998] and Japan Society for the Promotion of Science (No. PS95785[1996]). (Manuscript received April 30, 1996, revised June 10, 1996)

\section{References}

(1) G. D. Mahan, L. M. Levinson and H. R. Philipp, J. Appl. Phys, 50(4), 2799-2812 (1979).

(2) L. M. Levinson and H. R. Philipp, Am. Cream. Soc. Bull, 65(4), 639-646 (1986).

(3) J. Wong, J. Appl. Phys, 47(11), 4971-4974 (1976).

(4) J. T. C. Kemenade and R. K. Ejinthoven, J. Appl. Phys, 50(2), 938-941 (1979).

(5) O. L. Krivanek and P. Williams, Appl. Phys. Lett, 34 (11), 805-806 (1979).

(6) Guangping Jia, A study on dimensional effect of $\mathrm{ZnO}$ varistor, MSC thesis, $\mathrm{Xi}^{\prime}$ an Jiaotong University, 1992.

Shengtao $\mathrm{Li}$ (Non-member) He was born in P. R. China in

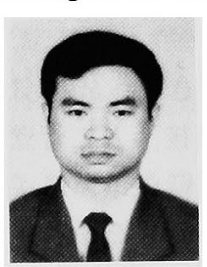

1963. He received B. Eng, M. Eng. and D. Eng degrees in electrical engineering from $\mathrm{Xi}^{\prime}$ an Jiaotong University, in 1983, 86 and 90 , respectively. He is an associate professor of School of Electrical Engineering, $\mathrm{Xi}^{\prime}$ an Jiaotong University.

Fuyi Liu (Non - member) She was born in P. R. China in

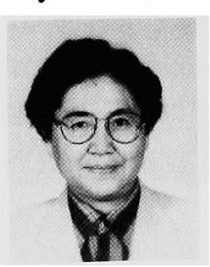

1937. She received B. Eng and M. Eng degrees in electrical engineering from $\mathrm{Xi}^{\prime}$ an Jiaotong University in 1960 and 64, respectively She is a professor of School of Electrical Engineering, $\mathrm{Xi}^{\prime}$ an Jiaotong University.

Gunagping Jia (Non-member) He was born in P. R. China

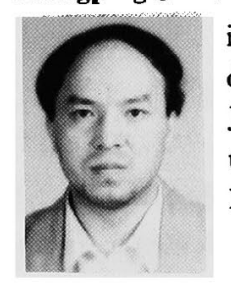
in 1964. He received E. Eng and M. Eng degrees in electrical engineering from $\mathrm{Xi}^{\prime}$ an Jiaotong University, in 1986 and 92 , respectively. He is now an engineer of $\mathrm{Xi}^{\prime}$ an Radio Factory No. 2

Yoshimichi Ohki (Member) Refer to Trans, IEE Japan, 116 -A, 545 (1996) 